\title{
View Updates in Stratified Disjunctive Databases*
}

\author{
John Grant ${ }^{2,4}$ \\ John Horty ${ }^{2,3}$ \\ Jorge Lobo ${ }^{5}$ \\ Jack Minker ${ }^{1,2}$ \\ ${ }^{1}$ Computer Science Department \\ ${ }^{2}$ Institute for Advanced Computer Studies \\ ${ }^{3}$ Philosophy Department \\ University of Maryland \\ College Park, MD 20742 \\ ${ }^{4}$ Department of Computer and Information Science \\ Towson State University \\ Towson, MD 21204
}

${ }^{5}$ Department of Electrical Engineering and Computer Science

The University of Illinois at Chicago

Chicago, Illinois 60680

*Research supported by the National Science Foundation under grant numbers IRI-8916059, IRI-8921591, IRI-9200898, and IRI-9210220 


\section{Introduction}

This paper is devoted to the problem of view updates in deductive databases. In this context, derived or intensional predicates correspond to the views of traditional relational databases. The view update problem is thus the problem of accomplishing the update of an intensional predicate by modifying appropriately the underlying relations in the extensional part of the database.

In the case of purely definite deductive databases, it is sometimes hard to see how the extensional relations should be modified to accomplish certain view updates. As an example, consider the simple database

$$
\begin{aligned}
& P(x) \leftarrow A(x) \\
& P(x) \leftarrow B(x),
\end{aligned}
$$

where $P$ is an intensional predicate, and $A$ and $B$ are extensional. Suppose we want to update this database with the information that $P(c)$. If we are restricted to definite clauses, there are only three plausible ways to do this: add $A(c)$, add $B(c)$, or add both $A(c)$ and $B(c)$. Each of the first two options seems arbitrary; the third results in an update that is too strong. If we allow disjunctive information into the database, however, we can accomplish the update by adding $A(c) \vee B(c)$; and this option appears intuitively to be correct.

We define here general algorithms for accomplishing view updates (both insertions and deletions) in disjunctive deductive databases; and we provide a semantic justification of the updates accomplished by these algorithms, making precise a sense in which they represent "minimal modifications" of the underlying database. We consider two kinds of updates: those involving the insertion or deletion of information into and from a disjunctive deductive database, and those involving the insertion or deletion of information into and from a stratified normal disjunctive database. Insertions into normal deductive databases may require the insertion of negative information. (Of course, a database cannot contain negative information explicitly; but it can yield such information through nonmonotonic reasoning techniques, such as closed world reasoning [7,6].)

There has recently been a good deal of work devoted to the view update problem in deductive databases. Fagin et al. [2] provide a semantic characterization of correctness that forms the basis of our own. That paper works with a fully expressive logical language, however, and so it cannot contain algorithms for computing the appropriate updates; in addition, it does not deal with negative information concluded through closed world reasoning. More recently, the problem has been investigated by Guessoum and Lloyd [4, 5]; but they limit their

treatment to definite databases, and so the algorithms they provide do not agree with the semantics of [2]. They also allow changes in the intensional database.

The work that is closest to our own is that of Rossi and Naqvi [8]. This work does deal with disjunctive information, and it provides algorithms; however, unlike the approach presented here, it does not insert the disjunctive information directly into the extensional database (which is supposed to remain definite), but keeps 
it aside in a filter that is then to be used in the process of query evaluation.

\section{The language}

A deductive database $D B$ is divided into an intensional component $I_{D B}$ and an extensional component $E_{D B} . L$ is the background language of the database, the predicates occurring in $L$ are partitioned into two sets: the $L_{I}$ predicates are intensional and the $L_{E}$ predicates are extensional. Those predicates occurring in $E_{D B}$ belong to $L_{E}$; the predicates occurring in the heads of $I_{D B}$ rules belong to $L_{I}$. For simplicity, constants are substituted for variables in the database, so we can deal with ground atoms rather than predicates; these atoms are said to be intensional or extensional depending on the kind of predicate they contain. There are no denials or integrity constraints.

Updates must involve modifications of the extensional database only; the rules are not modified. Hence we assume, without further elaboration, that a view update to DB does not change $I_{D B}$. We also assume that $E_{D B}$ does not contain redundant information, such as $A \leftarrow$ and $A \vee B \leftarrow$. Hence we include a subsumption elimination step in our algorithms. This is not a crucial point, however, and subsumption elimination may be omitted in all cases. Since in our case there are no variables or function symbols, subsumption checking involves only checking for substrings.

In Section 3 we focus on a special kind of disjunctive database: the intensional part consists of definite rules and the extensional part consist of positive clauses which may be disjunctive. The definition is given below. In Section 4 we extend Definition 2.1 to obtain stratified disjunctive databases.

Definition 2.1 Let $L$ be a function-free first order language. Let $D B$ be a first order theory in $L$. Then $D B$ is called a disjunctive database iff

1. $D B=E_{D B} \cup I_{D B}$ and $E_{D B} \cap I_{D B}=\emptyset$

2. $C \in E_{D B}$ iff $C$ is a positive ground clause.

3. $C \in I_{D B}$ iff $C$ is a clause of the form $A \leftarrow B_{1}, \ldots, B_{m}$, with $A, B_{1}, \ldots, B_{m}$ ground atoms and $m>0$.

4. The predicate symbols occurring in $E_{D B}$ do not appear in the head of any rule in $I_{D B}$.

\section{Updating disjunctive databases}

This section describes techniques for updating disjunctive databases. Here, the algorithms for insertion and deletion are described in terms of proof trees constructed with SLD-derivations. 


\section{$3.1 \quad$ Insertions}

We first define a special kind of SLD-tree, as follows.

Definition 3.1 Let $L$ be a function-free first order language. Let $D B$ be a disjunctive database in L. Let $P$ be an atom (in this case, $P$ is ground) such that $P$ is intensional. Assume $R$ to be a computation rule that only selects atoms from $L_{I}$. A restricted SLD-tree for the goal $G=\leftarrow P$ given $D B$ is a tree satisfying the following conditions:

1. The root of the tree is $G$.

2. Each node in the tree is a (possibly empty) goal.

3. A goal $\gamma$ is a child of a node $\Lambda$ if $\gamma$ can be SLD-derived from $\Lambda$ via $R$.

A restricted SLD-tree may contain infinite branches. However, since variables are not included in the goals, it is possible to check for repeated ancestors of a selected literal before expanding it.

Example 3.1 Let $D B$ be the disjunctive database:

$$
\begin{aligned}
& P \leftarrow A, B \\
& P \leftarrow E \\
& P \leftarrow Q, C \\
& Q \leftarrow A, D \\
& A \vee B \vee C \leftarrow
\end{aligned}
$$

Let $\{A, B, C, D, E\}$ be the set of extensional atoms and $\{P, Q\}$ be the set of intensional atoms. The restricted SLD-tree for $\leftarrow P$ in $D B$ is the following:

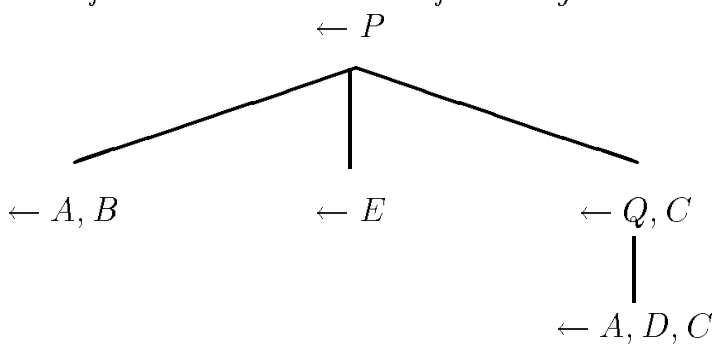

A statement $P$ is a logical consequence of $D B$ if any of the conjunctions of atoms in the leaves in a restricted SLD-tree for the goal $G=\leftarrow P$ is true in $D B$. Take, for example the leftmost leaf in Example 3.1 . If $A$ and $B$ are true in $D B$, then $P$ is also true in $D B$. Since it is desirable to "minimize" the changes to $D B$, the insertion algorithm needs to consider the weakest formula that achieves the insertion. In the example, this formula is given by the disjunction of these leaves: $(A \wedge B) \vee(E) \vee(A \wedge D \wedge C)$. 
Since we are limited to modifying only the extensional part of the disjunctive database, there are some updates that are not possible.

Example 3.2 Let $D B$ be the disjunctive database:

$$
P-Q, C
$$

Let $\{C\}$ be the set of extensional atoms and $\{P, Q\}$ the set of intensional atoms. The insertion of $P$ into $D B$ requires the insertion of $Q$. The only possible modification to the extensional part of $D B$ is to insert $C$. There is no way to insert $Q$.

Definition 3.2 Let $D B$ be a disjunctive database such that $D B \not P$. An insertion of an atom $P$ is a possible insertion into $D B$ iff there exists a disjunctive database $D B^{\prime}$ such that $D B^{\prime} \vdash P$.

Algorithm 1 (Insertion of an intensional atom $P$ into a disjunctive database - Insertion 1)

Given an atom $P$ and a disjunctive database $D B$ such that $D B \nvdash P$, the algorithm computes a database $D B^{\prime}$ such that $D B^{\prime} \vdash D B \wedge P$ whenever the insertion is possible.

1. Let $D B^{\prime}=D B$.

2. Construct a restricted SLD-tree for $\leftarrow P$ from $D B$.

3. Let $\leftarrow F_{1}, \ldots, \leftarrow F_{n}$ be the leaf nodes containing only conjunctions of extensional atoms. If $n=0$ the insertion fails. Otherwise, construct the conjunctive normal form of $F_{1} \vee \cdots \vee F_{n}$, writing it as $C_{1} \wedge \cdots \wedge C_{m}$, where subsumed clauses are omitted from the conjunctive normal form.

4. For each $C_{i}, 1 \leq i \leq m$, insert $C_{i}$ into $D B^{\prime}$.

5. Delete all subsumed clauses from $D B^{\prime}$.

Example 3.3 From the restricted SLD-tree of Example 3.1 we obtain $F_{1}=A \wedge B, F_{2}=E$ and $F_{3}=A \wedge D \wedge C$. Then, $D B \vdash P$ if $D B \vdash F_{1} \vee F_{2} \vee F_{3}$. The conjunctive normal form of $F_{1} \vee F_{2} \vee F_{3}$ is $C_{1} \wedge C_{2} \wedge C_{3}$, where $C_{1}=A \vee E, C_{2}=B \vee D \vee E, C_{3}=B \vee C \vee E$. Then, the updated database $D B^{\prime}$ is:

$P \leftarrow A, B$

$P \leftarrow E$

$P \leftarrow Q, C$

$Q \leftarrow A, D$

$A \vee B \vee C \leftarrow$

$A \vee E \leftarrow$ 
$B \vee C \vee E \leftarrow$

$B \vee D \vee E \leftarrow$

In order to justify this algorithm, we must show first that it actually accomplishes the desired insertion.

Lemma 3.1 Let $D B$ be a disjunctive database in L. Let $P$ be an atom in $L_{I}$ and assume that the insertion of $P$ into $D B$ is possible, and $D B \forall P$. Then, if $D B^{\prime}$ is the deductive database constructed by Algorithm 1 , $D B^{\prime} \vdash D B \wedge P$.

Proof: $D B^{\prime} \vdash D B$ follows from Steps 1, 4, and 5 . Now, let $M$ be a model of $D B^{\prime}$. By construction, each $C_{i}$, $1 \leq i \leq m$, as developed by Algorithm 1 , is true in $M$. Hence $F_{1} \vee \cdots \vee F_{n}$ is true in $M$. By the soundness of SLD-derivations and the intensional parts of rules of $D B^{\prime}, P$ is true in $M$.

But of course, this is not enough; in addition, we must require that the insertion should modify the original database as little as possible, where this can be defined as follows.

Definition 3.3 Let $D B$ be a disjunctive database in $L$. Let $P$ be an atom in $L_{I}$ and assume $D B \forall P$. $A$ minimal insertion of $P$ into $D B$ is a minimal $D B^{\prime}$ such that $D B^{\prime} \vdash D B \wedge P$. That is, there is no database $D B^{\prime \prime}$ such that $D B^{\prime} \vdash D B^{\prime \prime} \vdash D B \wedge P$ and $D B^{\prime \prime} \neq D B^{\prime}$.

As the following lemma shows, Algorithm 1 does in fact produce a minimal insertion.

Lemma 3.2 Let $D B$ be a disjunctive database in L. Let $P$ be an atom in $L_{I}$ and assume that the insertion of $P$ into $D B$ is possible, and $D B \forall P$. Let $D B^{\prime}$ be the deductive database that is obtained from Algorithm 1 after inserting $P$. If $D B^{\prime} \vdash D B^{\prime \prime} \vdash D B$ and $D B^{\prime \prime} \forall D B^{\prime}$ then $D B^{\prime \prime} \forall P$.

Proof: By construction, $D B^{\prime}=D B \cup\left\{C_{i} \in\left\{C_{1}, \ldots, C_{m}\right\} \mid D B \forall C_{i}\right\}$, where the $C_{i}$ are obtained in step 4 of Algorithm 1. Therefore, since $D B^{\prime \prime} \forall D B^{\prime}$ and $D B^{\prime \prime} \vdash D B, \exists i, 1 \leq i \leq m$, such that $D B^{\prime \prime} \forall C_{i}$. Hence, $D B^{\prime \prime} \nvdash F_{1} \vee \cdots \vee F_{n}$. Hence, $D B^{\prime \prime} \forall P$.

Unfortunately, however, the minimality condition alone does not imply uniqueness, and so it cannot be used as a criterion to justify the insertion algorithm. This should be obvious already from the example used in the introduction; there, the addition of either $A(c)$ or $B(c)$ yields a minimal modification of the database. In order to force a unique result, we require not only that the update should be minimal, but also that it must yield the weakest modification of the database, in the following sense. 
Definition 3.4 Let $D B_{1}$ and $D B_{2}$ be disjunctive databases. $D B_{1}$ is weaker than $D B_{2}$ iff $D B_{2} \vdash D B_{1}$. Let $\mathcal{D B}$ be a set of disjunctive databases. $D B$ is the weakest disjunctive database in $\mathcal{D B}$ iff for any other disjunctive database $D B^{\prime}$ in $\mathcal{D B}, D B$ is weaker than $D B^{\prime}$.

The following theorem shows that Algorithm 1 constructs the weakest of the minimal disjunctive databases that accomplish the desired update.

Theorem 3.3 Let $D B$ be a disjunctive database in L. Let $P$ be an atom in $L_{I}$ and assume that the insertion of $P$ into $D B$ is possible, and $D B \nvdash P$. Let $D B^{\prime}$ be the disjunctive database that is obtained from Algorithm 1 after inserting $P$. Then, $D B^{\prime}$ is the weakest disjunctive database $D B^{\prime \prime}$ such that $D B^{\prime \prime} \vdash D B \wedge P$.

Proof: Let $D B^{\prime \prime} \vdash D B \wedge P$. If $F \in D B$ then $D B^{\prime \prime} \vdash F$. Now suppose that $F \in\left(D B^{\prime}-D B\right)$. Then $F=C_{i}$, for some $i, 1 \leq i \leq m$, where $C_{i}$ is a clause formed by Algorithm 1. Since $D B^{\prime \prime} \vdash P$, by the construction of the $C_{i} \mathrm{~s}$, and the completeness of SLD-resolution, $D B^{\prime \prime} \vdash C_{i}$.

Another interesting characterization of the weakest minimal insertion is given by the disjunction of all minimal insertions.

Definition 3.5 Let $D B_{1}, \cdots, D B_{n}$ be disjunctive databases with identical intensional portion $I_{D B}$. Then

$$
\bigvee_{i=1}^{n} D B_{i}=\left\{C_{1} \vee \cdots \vee C_{n} \mid C_{i} \in E_{D B_{i}}, 1 \leq i \leq n\right\} \cup I_{D B}
$$

Theorem 3.4 Let $D B$ be a disjunctive database in L. Let $P$ be an atom in $L_{I}$ and assume that the insertion of $P$ into $D B$ is possible, and $D B \nvdash P$. Let $D B^{\prime}$ be the disjunctive database that is obtained from Algorithm 1 after inserting $P$. Let $D B_{1}, \ldots, D B_{n}$ be all the minimal updates that insert $P$ into $D B$. Then $D B^{\prime}$ is logically equivalent to $\bigvee_{i=1}^{n} D B_{i}$.

Proof: Since $D B^{\prime}$ is the weakest insertion, $D B_{i} \vdash D B^{\prime}$, therefore, $\bigvee_{i=1}^{n} D B_{i} \vdash D B^{\prime}$. Conversely, since $D B^{\prime}$ is a minimal insertion, $D B^{\prime}$ is one of the $D B_{i}$. Therefore, $D B^{\prime} \vdash \bigvee_{i=1}^{n} D B_{i}$.

This final characterization of the appropriate result of insertion updates can be derived easily from that of Fagin et al. [2]; Theorem 3.3 thus shows how the characterization can be reached through a slightly different route.

\subsection{Deletions}

We now present the algorithm for updating disjunctive databases by deleting information from the database. We note that in a definite database when an intensional atom $P$ is deleted, $\neg P$ becomes true in the updated 
database. The reason for this is that a definite database has a unique minimal model where either $P$ or $\neg P$ is true. However, a disjunctive database may have several minimal models. The deletion of $P$ may be accomplished by deleting $P$ from some but not all of the minimal models. Hence the deletion of $P$ does not necessarily make $\neg P$ true.

Definition 3.6 Let $D B$ be a disjunctive database such that $D B \vdash P$. A deletion of an atom $P$ from $D B$ results in a disjunctive database $D B^{\prime}$ such that $D B^{\prime} \nvdash P$.

Note that the deletion of an atom, unlike insertion, is always possible.

Algorithm 2 (Deletion of an intensional atom P from a disjunctive database - Deletion 1)

Given an atom $P$ and a disjunctive database $D B$ such that $D B \vdash P$. The algorithm computes a database $D B^{\prime \prime}$ such that $D B^{\prime \prime} \forall P$.

1. Construct a restricted $S L D-$ tree for $\leftarrow P$ from $D B$.

2. Let $\leftarrow F_{1}, \ldots, \leftarrow F_{n}$ be the leaf nodes containing only conjunctions of extensional atoms. Construct the conjunctive normal form of $F_{1} \vee \cdots \vee F_{n}$, writing it as $C_{1} \wedge \cdots \wedge C_{m}$, where subsumed clauses are omitted in the conjunctive normal form.

3. Let $S_{i}$ be the sets of clauses in $D B$ which subsume $C_{i}, 1 \leq i \leq m$. For each $j, 1 \leq j \leq m$ such that there is no $i, i \neq j$ and $1 \leq i \leq m$, such that $S_{i} \subset S_{j}$, obtain $D B_{j}=\left(D B-S_{j}\right)$. Without loss of generality assume that the remaining $D B_{j}$ are $D B_{1}, \ldots, D B_{k}$.

4. Let $D B^{\prime \prime}$ be $\bigvee_{j=1}^{j=k} D B_{j}$.

5. Delete all subsumed clauses from $D B^{\prime \prime}$.

Example 3.4 Let $D B$ be the disjunctive database:

$$
\begin{aligned}
& P \leftarrow A, B \\
& P \leftarrow E \\
& P \leftarrow Q, C \\
& Q \leftarrow A, D \\
& A \leftarrow \\
& B \vee D \leftarrow \\
& B \vee E \leftarrow
\end{aligned}
$$

$I_{D B}$ is the same as in Example 3.1. Hence the restricted $S L D$-tree for $\leftarrow P$ is the same, so $C_{1}=A \vee E$, 
$C_{2}=B \vee D \vee E, C_{3}=B \vee C \vee E$. Hence, $S_{1}=\{A\}, S_{2}=\{B \vee D, B \vee E\}, S_{3}=\{B \vee E\}$. Since $S_{3} \subseteq S_{2}, S_{2}$ is eliminated, leaving $S_{1}$ and $S_{3}$. From step 3 we obtain the disjunctive databases $D B_{1}=I_{D B} \cup\{B \vee D, B \vee E\}$ and $D B_{3}=I_{D B} \cup\{A, B \vee D\}$. Then, $D B^{\prime \prime}$ is:

$$
\begin{aligned}
& I_{D B} \cup \\
& \{B \vee D \leftarrow \\
& A \vee B \vee E \leftarrow\} .
\end{aligned}
$$

The following lemma shows that Algorithm 2 accomplishes the desired deletion.

Lemma 3.5 Let $D B$ be a deductive database in $L$. Let $P$ be an atom in $L_{I}$ and assume $D B \vdash P$. Then the deductive database $D B^{\prime \prime}$ constructed by Algorithm 2 does not imply $P$. That is, $D B^{\prime \prime} \forall P$.

Proof: By construction and the soundness and completeness of SLD-resolution for all $i, 1 \leq i \leq m,\left(D B-S_{i}\right) \not$ $C_{i}$, where $C_{i}$ is obtained from Algorithm 2. Therefore $\left(D B-S_{i}\right) \nvdash P$. Hence $D B^{\prime \prime} \nvdash P$.

In the previous case of inserting information, we required that the update should modify the original database as little as possible; this led us to present in Definition 3.3 the concept of a minimal insertion. In the case of deletions, the counterpart idea of a minimal modification can be defined as a maximal subset of the original database that accomplishes the desired deletion.

Definition 3.7 Let $D B$ be a disjunctive database in $L$. Let $P$ be an atom in $L_{I}$ and assume $D B \vdash P$. $A$ minimal deletion of $P$ from $D B$ is a maximal subset $D B_{i}$ of $D B$ that does not imply $P$. That is, $D B_{i} \subseteq D B$, $D B_{i} \nvdash P$ and for any other disjunctive database $D B^{\prime} \subseteq D B$ such that $D B_{i} \subset D B^{\prime}, D B^{\prime} \vdash P$.

Again, however, there may be several such minimal deletions; and so, in accordance with the views of Fagin et al.[2], it seems that a semantically correct algorithm should yield a result equivalent to their disjunction. The following theorem shows that Algorithm 2 is correct in this sense.

Theorem 3.6 Let $D B$ be a disjunctive database in $L$. Let $P$ be an atom in $L_{I}$ and assume that $D B \vdash P$. Let $D B^{\prime \prime}$ be the disjunctive database that is obtained from Algorithm 2 by deleting $P$. Let $D B_{1}, \ldots, D B_{n}$ be all the minimal updates that delete $P$ from $D B$. Then $D B$ "is logically equivalent to $\bigvee_{i=1}^{n} D B_{i}$.

Proof: The proof follows from the observation that the minimal deletions of $P$ are precisely the $\left(D B-S_{i}\right)$ obtained in step 3 of Algorithm 2. 
As it turns out, there is an interesting asymmetry between insertion and deletion. In the case of insertion, the disjunction of minimal insertions is actually equivalent to a particular one of the minimal insertions; but in the case of deletion, the disjunction of minimal deletions need not itself lie among the minimal deletions.

A peculiar property of the deletion algorithm is that logically equivalent databases that are syntactically different can be transformed into non-equivalent databases after the deletion of an atom from the database. Consider, for example the disjunctive database $D B$ in Example 3.4. Extend $D B$ with the extensional fact $A \vee B$. The new disjunctive database is equivalent to $D B$ since $A$ subsumes $A \vee B$. The deletion of $P$ from this new database using Algorithm 2 results in the disjunctive database:

$$
\begin{aligned}
D B^{\prime}= & I_{D B} \cup \\
& \{B \vee D ; \\
& A \vee B\} .
\end{aligned}
$$

$D B^{\prime \prime}$ from Example 3.4 is weaker than this database. The minimality condition does not guarantee the preservation of equivalence after updating equivalent databases. In the case of insertion, in addition to minimality, the weakness condition was used to select the best update. The weakest insertion was the insertion selected. A similar condition can be imposed on deletion. We call it the strongness condition.

Definition 3.8 Let $D B_{1}$ and $D B_{2}$ be disjunctive deductive databases. $D B_{1}$ is stronger than $D B_{2}$ iff $D B_{1} \vdash$ $D B_{2}$. Let $\mathcal{D B}$ be a set of disjunctive databases. $D B$ is the strongest disjunctive database in $\mathcal{D B}$ iff for any other disjunctive deductive database $D B^{\prime}$ in $\mathcal{D B}, D B$ is stronger than $D B^{\prime}$.

In general, there is no strongest disjunctive database that does not imply an atom $P$ and is weaker than $D B$. Moreover, there is not a direct correspondence between minimal and strong deletions. The following algorithm non-deterministically selects a disjunctive database $D B^{\prime}$ that 1) accomplishes the deletion, 2) is weaker than $D B$ and 3 ) is stronger or incomparable to any other disjunctive database containing $I_{D B}$ that does not imply $P$.

Algorithm 3 (Deletion of an intensional atom P from a disjunctive database - Deletion 2)

Given an atom $P$ and a disjunctive database $D B$ such that $D B \vdash P$. The algorithm computes a database $D B^{\prime}$ such that $D B^{\prime} \forall P$.

1. Construct a restricted $S L D-$ tree for $\leftarrow P$ from $D B$.

2. Let $\leftarrow F_{1}, \ldots, \leftarrow F_{n}$ be the leaf nodes containing only conjunctions of extensional atoms. Construct the conjunctive normal form of $F_{1} \vee \cdots \vee F_{n}$, writing it as $C_{1} \wedge \cdots \wedge C_{m}$ where subsumed clauses are omitted.

3. Let $S_{i}$ be the sets of clauses in DB which subsume $C_{i}, 1 \leq i \leq m$. Choose $j, 1 \leq j \leq m$ such that there is no $i, i \neq j$ and $1 \leq i \leq m, S_{i} \subset S_{j}$. Delete the set $S_{j}$ from $D B$. 
4. For each clause $C \in S_{j}$, form all disjunctions $C \vee Q$ where $Q$ is an extensional atom that does not appear in $C$.

5. Add to $D B$ all clauses $C \vee Q$ which do not subsume $C_{j}$. Call the resulting database $D B^{\prime}$.

Example 3.5 Using the same disjunctive deductive database as in Example 3.4, there are two answers for the deletion of $P$ using Algorithm 3 depending on the choice of $S_{1}$ or $S_{3}$ (see Example 3.4). These are (omitting the intensional part which is the same in all cases):

$$
\begin{array}{rr}
\text { 1) }\{A \vee B ; & \text { 2) }\{A ; \\
A \vee C ; & B \vee D\} . \\
A \vee D ; & \\
B \vee D ; & \\
B \vee E\} . &
\end{array}
$$

The following lemma shows that Algorithm 3 accomplishes the deletion.

Lemma 3.7 Let $D B$ be a disjunctive deductive database in $L$. Let $P$ be an intensional atom in $L_{I}$. Assume that $D B \vdash P$. Then, the deductive database $D B^{\prime}$, constructed by Algorithm 3 does not imply $P$. That is, $D B^{\prime} \forall P$.

Proof: By construction, $D B^{\prime} \not C_{j}$, where $C_{j}$ is derived in Algorithm 3. Therefore, $D B^{\prime} \forall C_{1} \wedge \cdots \wedge C_{m}$ and hence $D B^{\prime} \forall P$.

Theorem 3.8 shows that there is no stronger deductive database than $D B^{\prime}$ that deletes $P$.

Theorem 3.8 Let $D B$ be a disjunctive deductive database in $L$. Let $P$ be an intensional atom in $L_{I}$. Assume $D B \forall P$. Let $D B^{\prime}$ be the deductive database that is obtained from Algorithm 3 after deleting $P$. Then, there is no deductive database $D B^{\prime \prime}$ stronger than $D B^{\prime}$ and weaker than $D B$ such that $D B^{\prime \prime} \forall P$.

Proof: Since the clauses added to the database in step 5 are logically implied by $D B, D B \vdash D B^{\prime}$. Now suppose that $D B \vdash D B^{\prime \prime} \vdash D B^{\prime}$ and assume $D B^{\prime} \nvdash D B^{\prime \prime}$. Let $C_{1} \wedge \cdots \wedge C_{m}$ be the conjunctive formula obtained in step 2 of Algorithm 3. Let $S_{j}$ be the set selected in step 3 for deletion. Since for all $i, i \neq j, D B^{\prime} \vdash C_{i}$, then $D B^{\prime \prime} \vdash C_{i}$, where $C_{i}$ is derived in Algorithm 3. But since $D B \vdash D B^{\prime \prime}$ and $D B^{\prime} \forall D B^{\prime \prime}$, by the construction, $D B^{\prime \prime} \vdash C_{j}$, so $D B^{\prime \prime} \vdash P$.

If we prefer to have a unique disjunctive database after a deletion as in the case of Algorithm 2, we can take the disjunction of all possible disjunctive databases obtained with Algorithm 3. This database is stronger than 
the disjunction of the minimal updated databases. A disadvantage of strong databases over minimal databases is that they are language dependent. In step 4, new disjunctions are formed to be included in the updated database, one disjunction for each extensional atom in the underlying language of the database. So the algorithm produces from two logical equivalent databases over different languages non-equivalent databases.

\section{Updating stratified disjunctive databases}

\subsection{Normal insertions}

In this section we present an algorithm to insert information into a subclass of normal disjunctive databases. A normal disjunctive database $D B$ is a disjunctive database in which the clauses in the $I_{D B}$ can be of the form $A \leftarrow L_{1}, \ldots, L_{m}$ with $A$ a ground atom, $L_{1}, \ldots, L_{m}$ ground literals (i.e. atoms and negated atoms) and $m \geq 0$. We consider insertions into normal databases that are stratified. The definitions of stratified databases, stratification, and the stratum of an atom are as defined in [1].

The insertion of an atom into a normal disjunctive database may require the "insertion" of negative information into the database. Consider, for example, a database $D B$ with $I_{D B}=\{P(x) \leftarrow \neg A(x)\}$. Assume that $A$ is an extensional predicate. The insertion of $P(a)$ into $D B$ must modify $D B$ into a new database $D B^{\prime}$ such that the new database implies $\neg A(a)$. Also, because of this duality between the insertion of atoms and negated atoms into a normal disjunctive database, the algorithm below can be used for both kinds of insertions, positive and negative.

Negative information can be derived from databases using nonmonotonic reasoning techniques, such as closed world reasoning. Because we are working with disjunctive databases, we use the generalized closed world assumption (GCWA), devised by Minker [6]. According to this rule, a ground formula $\neg F$ is derivable from a disjunctive database $D B$ if $F$ is false in all minimal models of that database. In that case, we say $G C W A(D B) \vdash \neg F$.

For the insertion algorithm into stratified databases we need to extend the definition of restricted SLD-trees to cover negation. The new trees will be called restricted stratified SLD-trees. We use normal goals where literals of the form not $(C)$ may appear. Restricted stratified SLD-trees are defined inductively on the stratum of the atom associated with the tree.

Definition 4.1 Let $D B$ be a stratified disjunctive database in $L$. Let $P$ be an atom (in this case, $P$ is ground) such that $P$ is intensional. Assume $R$ to be a computation rule that only selects literals with atoms from $L_{I}$. A restricted stratified SLD-tree for the goal $G=\leftarrow P$ given $D B$ is a restricted $S L D$-tree for $\leftarrow P$ if the stratum of $P$ is 1. Otherwise, assume all restricted stratified SLD-trees for atoms in a stratum less than $n$ are defined and 
let the stratum of $P$ be $n$. A restricted stratified SLD-tree for $\leftarrow P$ is a tree satisfying the following conditions:

1. The root of the tree is $G$.

2. Each node in the tree is a (possibly empty) goal.

3. Let $L_{i}$ be the selected literal in the node $\Lambda=\leftarrow L_{1}, \ldots, L_{i-1}, L_{i}, L_{i+1}, \ldots, L_{k}$.

(a) If $L_{i}$ is positive, a normal goal $\gamma$ is a child of $\Lambda$ if $\gamma$ is SLD-derived from $\Lambda$ via $R$.

(b) If $L_{i}=\neg Q$ is negative, let $\leftarrow F_{1}, \ldots, \leftarrow F_{n}$ be the leaf nodes in the restricted stratified SLD-tree for $\leftarrow Q$. Construct the conjunctive normal form of $F_{1} \vee \cdots \vee F_{n}$, writing it as $C_{1} \wedge \cdots \wedge C_{m}$. A normal goal $\leftarrow L_{1}, \ldots, L_{i-1}, \gamma, L_{i+1}, \ldots, L_{k}$ is a child of $\Lambda$ iff $\gamma$ is the conjunction of literals obtained from $\neg C_{i}$, from some $C_{i}, 1 \leq i \leq m$.

Example 4.1 Let $D B$ be the stratified disjunctive database:

$$
\begin{aligned}
& Q \leftarrow A, \neg R \\
& R \leftarrow B, C \\
& R \leftarrow A, S \\
& S \leftarrow B, D \\
& A \vee C \leftarrow \\
& A \vee D \leftarrow
\end{aligned}
$$

The conjunctive normal form obtained from the restricted stratified SLD-tree for $\leftarrow R$ (in this case just restricted $S L D$-tree $)$ is $B \wedge(C \vee A) \wedge(C \vee D)$. Therefore, the restricted stratified SLD-tree for $\leftarrow Q$ is:

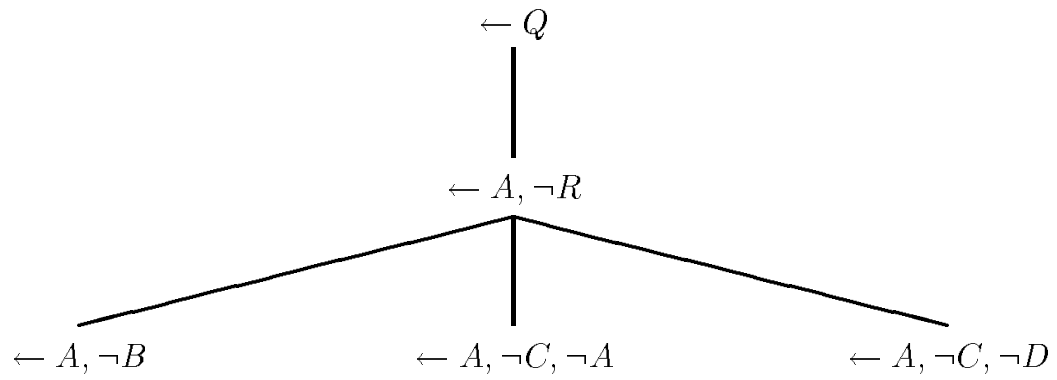

Algorithm 4 (Insertion of an intensional atom $P$ into a stratified disjunctive database - Insertion 2)

Suppose that $P$ is an intensional atom, and let $D B$ be a stratified disjunctive database such that $G C W A(D B) H$ $P$. The algorithm computes a database $D B^{\prime}$ such that $G C W A\left(D B^{\prime}\right) \vdash P$.

1. Let $\mathcal{M}$ be the minimal models of $E_{D B}$, the extensional component of $D B$.

2. Construct a restricted stratified $S L D-$ tree for $\leftarrow P$ from $D B$. 
3. Let $\leftarrow F_{1}, \ldots, \leftarrow F_{n}$ be the leaf nodes containing only conjunctions of (possibly negated) extensional atoms. If $n=0$ the insertion fails. Otherwise, construct the conjunctive normal form of $F_{1} \vee \cdots \vee F_{n}$, writing it as $C=C_{1} \wedge \cdots \wedge C_{m}$, where subsumed clauses are omitted from the conjunctive normal form.

4. If $E_{D B} \cup\{C\}$ is consistent then order $C$ by placing positive clauses first and negative clauses last. For each $C_{j}, 1 \leq j \leq m, d o$

(a) If $C_{j}$ is a positive clause, $C_{j}=A_{1} \vee \cdots \vee A_{l}$ then:

For each $M \in \mathcal{M}$ such that for all $A_{i}, 1 \leq i \leq l, A_{i} \notin M$ let $M_{i}=M \cup\left\{A_{i}\right\}, 1 \leq i \leq l$, and let $\mathcal{M}=(\mathcal{M}-\{M\}) \cup\left\{M_{i} \mid 1 \leq i \leq l\right\}$.

Eliminate from $\mathcal{M}$ every $M$ for which there exists $M^{\prime} \in \mathcal{M}$, such that $M^{\prime} \subset M$.

(b) If $C_{j}$ is a mixed clause, $C_{j}=A_{1} \vee \cdots \vee A_{l} \vee \neg E_{1} \vee \cdots \vee \neg E_{s}$ then:

For each $M \in \mathcal{M}$ such that for all $A_{i}, 1 \leq i \leq l, A_{i} \notin M$ and for all $E_{k}, 1 \leq k \leq s, E_{k} \in M$ let $M_{i}=M \cup\left\{A_{i}\right\}, 1 \leq i \leq l$, and let $\mathcal{M}=(\mathcal{M}-\{M\}) \cup\left\{M_{i} \mid 1 \leq i \leq l\right\}$.

Eliminate from $\mathcal{M}$ every $M$ for which there exists $M^{\prime} \in \mathcal{M}$, such that $M^{\prime} \subset M$.

This step must be repeated until there is a complete pass through all the mixed clauses which causes no change to $\mathcal{M}$.

(c) If $C_{j}$ is a negative clause, $C_{j}=\neg E_{1} \vee \cdots \vee \neg E_{l}$ then:

For each $M \in \mathcal{M}$ such that for all $E_{i}, 1 \leq i \leq l, E_{i} \in M$ eliminate $M$ from $\mathcal{M}$.

5. Build $D B^{\prime}$ from the new $\mathcal{M}$.

(Comment: If the DB is represented by using model trees as discussed in [3], it is not necessary to reconstruct all the clauses in $\left.E_{D B^{\prime}}\right)$.

6. Else ( $E_{D B} \cup\{C\}$ is inconsistent)

(a) Construct all $D B_{i}$ such that each $E_{D B_{i}}$ is a maximal subset of $\operatorname{Cn}\left(E_{D B}\right)$ (the set of positive logical consequences of $E_{D B}$ ) with the property that $E_{D B_{i}} \cup\{C\}$ is consistent. (This can be done by a process where first single elements of $\mathrm{Cn}\left(E_{D B}\right)$ are omitted to obtain $E_{D B_{j}}$ and the consistency of $E_{D B_{j}} \cup\{C\}$ is checked; when the latter is inconsistent, the process is iterated with $E_{D B_{j}}$ substituted for $\operatorname{Cn}\left(E_{D B}\right)$; the maximal sets $E_{D B_{i}}$ obtained this way are retained. The consistency of $E_{D B_{j}} \cup\{C\}$ can be checked by doing all possible resolutions using the elements of $E_{D B_{j}}$ and $C_{i}$ [the clauses in $C$ ]; the empty clause indicates inconsistency.) At the end, subsumed clauses are omitted from DB. Assume without loss of generality that these are $D B_{1}, \ldots, D B_{k}$.

(b) For each $D B_{i}, 1 \leq i \leq k$, apply steps 4 and 5 to obtain $D B_{i}^{\prime}$. 
(c) Let $D B^{\prime}=\bigvee_{i=1}^{k} D B_{i}^{\prime}$.

The next example illustrates the case where $D B^{\prime}$ is obtained using Steps 4 and 5 .

Example 4.2 Let $D B$ be the stratified disjunctive database:

$$
\begin{aligned}
& P \leftarrow \neg A, C, R \\
& P \leftarrow \neg D, R \\
& R \leftarrow B \\
& R \leftarrow E \\
& A \vee B \leftarrow \\
& A \vee D \leftarrow \\
& C \vee D \leftarrow \\
& C \vee E \leftarrow
\end{aligned}
$$

The clauses obtained by step 3 after the construction of the restricted stratified $S L D$-tree for $\leftarrow P$ are $C_{1}$ : $\neg A \vee \neg D, C_{2}: C \vee \neg D, C_{3}: B \vee E$.

The minimal models of the extensional database of $D B$ are $M M\left(E_{D B}\right)=\{\{A, C\},\{A, D, E\},\{B, D, E\}$, $\{B, C, D\}\}$. In this case $E_{D B}$ is consistent with the $C_{i}$ s. The algorithm first modifies $M M\left(E_{D B}\right)$ to make $C_{3}$ true. This produces the new set of minimal models $\{\{A, B, C\},\{A, C, E\},\{A, D, E\},\{B, D, E\},\{B, C, D\}\}$. Next, the set of minimal models is modified to make $C_{2}$, true. The algorithm produces the set $\{\{A, B, C\},\{A, C, E\}$, $\{A, C, D, E\},\{B, C, D, E\},\{B, C, D\}\}$. The models $\{A, C, D, E\}$ and $\{B, C, D, E\}$ are removed since they are not minimal. Finally, $C_{1}$ is true in the three remaining models. Then, the minimal models of the new extensional database are $M M\left(E_{D B^{\prime}}\right)=\{\{A, B, C\},\{A, C, E\},\{B, C, D\}\}$. Building the extensional database from these models we obtain:

$A \vee B \leftarrow$

$A \vee D \leftarrow$

$C \leftarrow$

$B \vee E \leftarrow$

The following example illustrates the case where step 6 must be used, that is, where $E_{D B} \cup\{C\}$ is inconsistent.

Example 4.3 Let $D B$ be the stratified disjunctive database:

$$
\begin{aligned}
& P \leftarrow R, \neg C, \neg D \\
& P \leftarrow A, \neg B, \neg D \\
& P \leftarrow B, \neg C, \neg A
\end{aligned}
$$




$$
\begin{aligned}
& R \leftarrow A \\
& R \leftarrow B \\
& S \leftarrow B, D \\
& A \vee B \leftarrow \\
& A \vee C \leftarrow \\
& D \leftarrow
\end{aligned}
$$

The conjunction of clauses obtained by step 3 after the construction of the restricted stratified SLD-tree for $\leftarrow P$ is $(A \vee B) \wedge(\neg B \vee \neg C) \wedge(\neg A \vee \neg D)$. This conjunct is inconsistent with $E_{D B}$. $E_{D B}$ has two minimal models $\{A, D\}$ and $\{B, C, D\}$. There are three maximal subsets of $C n\left(E_{D B}\right)$ consistent with the conjunction $C_{1} \wedge C_{2} \wedge C_{3}$ : $E_{D B_{1}}=\{A \vee B, A \vee C, A \vee D, B \vee D\}, E_{D B_{2}}=\{A \vee B, A \vee C, A \vee D, C \vee D\}$ and $E_{D B_{3}}=\{A \vee B, D\}$. Applying the insertion to each of these databases we obtain $E_{D B_{1}}^{\prime}=\{A, B\}, E_{D B_{2}}^{\prime}=\{A, C\}$ and $E_{D B_{3}}^{\prime}=\{B, D\}$. The disjunction of these three new databases together with the rules produces $D B^{\prime}=I_{D B} \cup\{A \vee B, A \vee D, B \vee C\}$.

The simple characterization of minimality from the treatment of positive updates does not apply directly to this more complicated context, where nonmonotonic reasoning is involved and insertions and deletions from and into the extensional database may be needed to accomplish the insertion of an intensional atom. However, there is a sense in which the insertion defined by Algorithm 4 is minimal. The new database first minimizes the deletion of positive conclusions from the original database and then, subject to that constraint, minimizes the addition of new positive conclusions. That is, insertions are preferred over deletions. As it turns out, this condition is enough to guarantee uniqueness. The following theorems characterize this property. The first theorem shows the minimality of $D B^{\prime}$ with respect to $D B$ and $C$.

Theorem 4.1 Let $C$ be the conjunction of the clauses obtained in step 3 of Algorithm 4 and assume that $E_{D B} \cup\{C\}$ is consistent. Then, for any positive clause $D, D B^{\prime} \vdash D$ iff $D B \cup\{C\} \vdash D$, where $D B^{\prime}$ is obtained in step 5 of Algorithm 4.

Proof: Since by the construction of $D B^{\prime}$ every (minimal) model of $D B^{\prime}$ is a model of $D B \cup\{C\}$, it follows that $D B \cup\{C\} \vdash D$ implies that $D$ is true in every model of $D B^{\prime}$. Therefore $D B^{\prime} \vdash D$. To prove the other direction by the contrapositive, let $D B \cup\{C\} \forall D$. Then, there must be a minimal model of $D B \cup\{C\}$, say $M=\left\{A_{1}, \ldots, A_{k}\right\}$ where $D$ is false. Let $D$ be $A_{k+1} \vee \cdots \vee A_{n} . M$ must be a model of $D B$. Since $M$ is also a model of $C$, by the construction of $D B^{\prime}$, if $M$ is a minimal model of $D B$ then $M$ remains a minimal model for $D B^{\prime}$. Hence, $D B^{\prime} \forall D$. Otherwise $M$ is a non-minimal model of $D B$. Therefore, there is $M^{\prime} \subset M$, say $M^{\prime}=\left\{A_{1}, \ldots, A_{i}\right\}$ such that $M^{\prime}$ is minimal model of $D B . M^{\prime}$ is not model of $D B \cup\{C\}$ because it is a proper subset of a minimal model of $D B \cup\{C\}$. Consider what happens to $M^{\prime}$ in step 4 of Algorithm 4 . For any $C_{j}$, 
part of $C$, that is true in $M^{\prime}$ the algorithm does nothing. Consider now some $C_{j}$ false in $M^{\prime}$. If $C_{j}$ is positive or mixed, then $M^{\prime}$ is expanded in a minimal way to satisfy $C_{j}$. The expansion, $M^{\prime \prime} \subseteq M$, since $M$ is a minimal model of $D B$. Hence $M^{\prime \prime}$ cannot be a model of $D$. In the case of negative $C_{j}$, since $M$ is a model of $C_{j}, M^{\prime}$ must already be a model of $C_{j}$. Therefore $M$ (or a subset of $M$ ) is a minimal model in $\mathcal{M}$ from which $D B^{\prime}$ is built. Hence, $D B^{\prime} \forall D$.

Theorem 4.1 suggests an alternative approach to obtaining the result of Step 4 since the algorithm obtains the logical consequences of $E_{D B} \cup\{C\}$ : Do all possible resolutions using elements of $E_{D B}$ and $C_{i}, 1 \leq i \leq m$. Obtain the set of all positive clauses at the bottom of the resolution trees and omit subsumed clauses from the set. The result is $E_{D B^{\prime}}$. This result shows an interesting connection between resolution and minimal model manipulation.

The following lemma is a technical result needed later to characterize the minimal models of $\bigvee_{i=1}^{k} D B_{i}$.

Lemma 4.2 Let $D B=\bigvee_{i=1}^{k} D B_{i}, \mathcal{M}$ the set of minimal models of $D B$, and $\mathcal{M}_{i}$ the set of minimal models of $D B_{i}, 1 \leq i \leq k$. Then $M \in \mathcal{M}$ iff $M \in \mathcal{M}_{i}$ for some $i, 1 \leq i \leq k$, and there is no $M^{\prime} \in \mathcal{M}_{j}$ for some $j$, $1 \leq j \leq k$, such that $M^{\prime} \subset M$.

\section{Proof:}

$(\Leftarrow)$ Suppose that $M \in \mathcal{M}_{i}$ for some $i, 1 \leq i \leq k$, and there is no $M^{\prime} \in \mathcal{M}_{j}$ for some $j, 1 \leq j \leq k$, such that $M^{\prime} \subset M$. Clearly $M$ is a model of $D B$. If $M$ is not a minimal model of $D B$ then there is $M^{\prime} \subset M$ a model of $D B$ which is minimal. This would require that $M^{\prime} \in \mathcal{M}_{j}$ for some $j, 1 \leq j \leq k$ contradicting the hypothesis.

$\Leftrightarrow$ We show the contrapositive. Suppose that $M \notin \mathcal{M}_{i}$ for any $i$. If $M$ is a superset of any $M^{\prime} \in \mathcal{M}_{i}$, then $M$ is not minimal since $M^{\prime}$ is a model of $D B$. On the other hand if for any set $M^{\prime} \in \mathcal{M}_{i}$, either $M \subset M^{\prime}$ or $M \bigcap M^{\prime} \neq M^{\prime}$ then there must exist a clause $D_{i}$ in each $D B_{i}$ such that $M$ does not model $D_{i}$. Then, $D_{1} \vee \cdots \vee D_{k}$ is not modeled by $M$ hence $M$ is not a model of $D B$.

Theorem 4.3 Let $D B$ be a stratified deductive database in $L$. Let $P$ be an atom in $L_{I}$ and assume $D B \nvdash P$. Then the deductive database $D B^{\prime}$, constructed by Algorithm 4 implies $P$. That is, $D B^{\prime} \vdash P$. Moreover, $D B^{\prime}=$ $\bigvee\left\{D B^{\prime \prime} \mid D B^{\prime \prime} \vdash P\right.$ and $\nexists D B^{\prime \prime \prime} \vdash P$, such that $\left.\operatorname{Cn}\left(E_{D B^{\prime \prime}}\right) \bigcap \operatorname{Cn}\left(E_{D B}\right) \subset \operatorname{Cn}\left(E_{D B^{\prime \prime \prime}}\right) \bigcap \operatorname{Cn}\left(E_{D B}\right)\right\}$.

Proof: Assume first that $E_{D B} \cup\left\{C_{1}, \ldots, C_{m}\right\}$ is consistent. Since, by Theorem $4.1 D B^{\prime}$ is equivalent to $\operatorname{Cn}(D B \cup$ $\left.\left\{C_{1}, \ldots, C_{m}\right\}\right)$ it follows that $D B^{\prime} \vdash P$. Now, since $E_{D B} \cup\left\{C_{1}, \ldots, C_{m}\right\}$ is consistent, again from Theorem 4.1 $\operatorname{Cn}\left(E_{D B^{\prime}}\right) \supseteq \operatorname{Cn}\left(E_{D B}\right)$. Therefore, $\nexists D B^{\prime \prime \prime} \vdash P$, such that $\operatorname{Cn}\left(E_{D B^{\prime}}\right) \bigcap \operatorname{Cn}\left(E_{D B}\right) \subset \operatorname{Cn}\left(E_{D B^{\prime \prime \prime}}\right) \cap \operatorname{Cn}\left(E_{D B}\right)$. Assume that there exists another $D B^{\prime \prime}$ such that $D B^{\prime \prime} \vdash P$ and $\nexists D B^{\prime \prime \prime} \vdash P$, such that $\operatorname{Cn}\left(E_{D B^{\prime \prime}}\right) \bigcap \operatorname{Cn}\left(E_{D B}\right) \subset$ $\operatorname{Cn}\left(E_{D B^{\prime \prime \prime}}\right) \bigcap \operatorname{Cn}\left(E_{D B}\right)$. Then it is also true that $\operatorname{Cn}\left(E_{D B^{\prime \prime}}\right) \supseteq \operatorname{Cn}\left(E_{D B}\right)$; otherwise we can choose $D B^{\prime}$ as $D B^{\prime \prime \prime}$ 
and show that $\operatorname{Cn}\left(E_{D B^{\prime \prime}}\right) \bigcap \operatorname{Cn}\left(E_{D B}\right) \subset \operatorname{Cn}\left(E_{D B^{\prime \prime \prime}}\right) \bigcap \operatorname{Cn}\left(E_{D B}\right)$. Let $M$ be a model of $D B^{\prime \prime}$. Then $M$ is also a model of $D B$ and a model of $\left\{C_{1}, \ldots, C_{m}\right\}$. But by Theorem 4.1,DB' contains all the possible positive resolvents of $D B$ with $\left\{C_{1}, \ldots, C_{m}\right\}$. Therefore, by the soundness and completeness of resolution, $M$ is also a model of $D B^{\prime}$. Therefore, $D B^{\prime \prime} \vdash D B^{\prime}$. Hence, the disjunction of all $D B^{\prime \prime}, \vee D B^{\prime \prime}$ is equivalent to $D B^{\prime}$.

If $E_{D B} \cup\left\{C_{1}, \ldots, C_{m}\right\}$ is inconsistent, consider a $D B_{i}$ as constructed in step 6a. By the argument above, for each $i, 1 \leq i \leq k, D B_{i}^{\prime} \vdash P$. That is, in every minimal model of each $D B_{i}^{\prime}, 1 \leq i \leq k$, each $C_{j}, 1 \leq j \leq m$, is true. By Lemma 4.2 and step $6 \mathrm{~b}$ the same is true for $D B^{\prime}$. Also, the constructions of the $D B_{i}^{\prime}$ assume that they play the role of $D B^{\prime \prime}$ in the statement of the theorem. Hence the result follows from the first part.

\subsection{Normal deletions}

Algorithm 5 (Deletion of an intensional atom from a stratified disjunctive database - Deletion 3) Suppose that $P$ is an intensional atom, and let $D B$ be a stratified disjunctive database such that $G C W A(D B) \vdash$ $P$. The algorithm computes a database $D B^{\prime}$ such that $G C W A(D B)^{\prime} \forall P$.

1. Let $\mathcal{M}$ be the set of minimal models of $E_{D B}$, the extensional component of $D B$.

2. Construct a restricted stratified $S L D-$ tree for $\leftarrow P$ from $D B$.

3. Let $F_{1}, \ldots, F_{n}$ be the leaf nodes containing only conjunctions of (possibly negated) extensional atoms. Construct the conjunctive normal form of $F_{1} \vee \cdots \vee F_{n}$, writing it as $C_{1} \wedge \cdots \wedge C_{m}$, where subsumed clauses are omitted from the conjunctive normal form.

4. Select a disjunction $C_{j}, 1 \leq j \leq m$. The selection is made giving preference to $C_{j}$ which satisfies step 5 or the first part of step 7 -if possible to minimize deletions.

5. If $C_{j}$ is a negative clause, $C_{j}=\neg E_{1} \vee \cdots \vee \neg E_{l}$ then:

For each $M \in \mathcal{M}$, let $e(M)$ be the number of atoms in $M$ from $\left\{E_{1}, \ldots, E_{l}\right\}$. Let $m=\max \{e(M)\}$ for all $M \in \mathcal{M}$. Select $M \in \mathcal{M}$ such that $e(M)=m$. Let $M^{\prime}=M \cup\left\{E_{1}, \ldots, E_{l}\right\}$. Let $\mathcal{M}=(\mathcal{M}-\{M\}) \cup\left\{M^{\prime}\right\}$. Eliminate from $\mathcal{M}$ any proper subset of $M^{\prime}$.

6. If $C_{j}$ is a positive clause, $C_{j}=A_{1} \vee \cdots \vee A_{l}$ then:

Let $M^{\prime}=H B_{E_{D B}}-\left\{A_{1}, \ldots, A_{l}\right\}$. If $M^{\prime}=\emptyset$ let $\mathcal{M}=\emptyset$; otherwise let $\mathcal{M}=\mathcal{M} \cup\left\{M^{\prime}\right\}$. Eliminate from $\mathcal{M}$ any proper superset of $M^{\prime}$.

7. If $C_{j}$ is a mixed clause, $C_{j}=A_{1} \vee \cdots \vee A_{l} \vee \neg E_{1} \vee \cdots \vee \neg E_{s}$ then:

If there exists a model in $\mathcal{M}$ that contains no $A_{i}, 1 \leq i \leq l$, then, for each $M \in \mathcal{M}$, let $e(M)$ be the 
number of atoms in $M$ from $\left\{E_{1}, \ldots, E_{s}\right\}$. Let $m=\max \{e(M)\}$ for all $M \in \mathcal{M}$. Select $M \in \mathcal{M}$ such that $e(M)=m$. Let $M^{\prime}=M \cup\left\{E_{1}, \ldots, E_{s}\right\}$. Let $\mathcal{M}=(\mathcal{M}-\{M\}) \cup\left\{M^{\prime}\right\}$.

Eliminate from $\mathcal{M}$ any proper subset of $M^{\prime}$.

Otherwise, let $M^{\prime}=H B_{D B}-\left\{A_{1}, \ldots, A_{l}\right\}$. Let $\mathcal{M}=\mathcal{M} \cup\left\{M^{\prime}\right\}$. Eliminate from $\mathcal{M}$ any proper superset of $M^{\prime}$.

8. Build $D B^{\prime}$ from the new $\mathcal{M}$.

Example 4.4 Let $D B$ be the stratified disjunctive database:

$$
\begin{aligned}
& P \leftarrow \neg Q \\
& Q \leftarrow \neg C, \neg D \\
& Q \leftarrow A, R \\
& R \leftarrow B, C \\
& R \leftarrow A, \neg D \\
& A \vee C \leftarrow \\
& B \vee D \leftarrow \\
& C \vee D \leftarrow
\end{aligned}
$$

Three conjuncts are obtained from the restricted SLD-tree for $\leftarrow P: C_{1}=C \vee D, C_{2}=\neg A \vee \neg B \vee \neg C$, and $C_{3}=\neg A \vee D$. The minimal models of the extensional part of the database are: $\mathcal{M}=\{\{A, D\},\{B, C\},\{C, D\}\}$.

Assume now that we want to delete $P$ from the database. Suppose we select $C_{2}$ for the deletion. The algorithm changes the model $\{B, C\}$ to $\{A, B, C\}$. Now $\mathcal{M}=\{\{A, D\},\{A, B, C\},\{C, D\}\}$. The effect on $D B$ is to add $A \vee D$.

Alternatively, if $C_{1}$ is selected, the new minimal model $\{A, B\}$ is added. The new $\mathcal{M}=\{\{A, B\},\{A, D\}$, $\{B, C\},\{C, D\}\}$. The effect on $D B$ is to delete $C \vee D$. Selecting $C_{3}$ has the same effect as selecting $C_{2}$.

The following lemma shows that Algorithm 5 accomplishes the desired deletion.

Lemma 4.4 Let $D B$ be a stratified deductive database in $L$. Let $P$ be an atom in $L_{I}$ and assume $D B \vdash P$. Then the deductive database $D B^{\prime}$, constructed by Algorithm 5 does not imply $P$. That is, $D B^{\prime} \forall P$.

Proof: The construction creates a database with a minimal model where the clause $C_{i}$ selected in step 4 is not true.

The next result shows in what sense the deletion is minimal. First of all, minimality is defined with respect to the $C_{j}$ that is chosen in step 4 . Observe that if step 5 or the first part in step 7 is the step executed to obtain 
$D B^{\prime}$ then $E_{D B^{\prime}} \vdash E_{D B}$. Hence, if insertions are preferred over deletions, a $C_{j}$ should be chosen for which step 5 or the first part of step 7 is applicable. Under these considerations there is no database $E_{D B^{\prime \prime}}$ that does not imply the chosen $C_{j}$ that is weaker than $E_{D B}$ but stronger than $E_{D B^{\prime}}$.

\section{Theorem 4.5}

1) There does not exist $D B^{\prime \prime}$, such that $D B^{\prime \prime} \forall C_{j}$ and $\operatorname{Cn}\left(E_{D B^{\prime}}\right) \cap \operatorname{Cn}\left(E_{D B}\right) \subset \operatorname{Cn}\left(E_{D B^{\prime \prime}}\right) \cap \operatorname{Cn}\left(E_{D B}\right)$.

2) There does not exists $D B^{\prime \prime}$, such that $D B^{\prime \prime} \forall C_{j}, \operatorname{Cn}\left(E_{D B^{\prime}}\right) \bigcap \operatorname{Cn}\left(E_{D B}\right)=\operatorname{Cn}\left(E_{D B^{\prime \prime}}\right) \bigcap \operatorname{Cn}\left(E_{D B}\right), E_{D B^{\prime}} \vdash$ $E_{D B^{\prime \prime}}, E_{D B^{\prime \prime}} \nvdash E_{D B^{\prime}}$

\section{Proof:}

Case 1. $C_{j}$ is negative, $\neg E_{1} \vee \cdots \vee \neg E_{k}$. In this case $E_{D B^{\prime}} \cap E_{D B}=E_{D B}$, hence statement 1 is satisfied automatically. For statement 2 , the existence of such an $E_{D B^{\prime \prime}}$ would require some atom missing from a minimal model of $E_{D B^{\prime}}$ which is impossible by the way the algorithm constructs $E_{D B^{\prime}}$.

Case 2. $C_{j}$ is mixed, $\neg E_{1} \vee \cdots \vee \neg E_{k} \vee A_{1} \vee \cdots \vee A_{l}$. There are two possibilities. First, if there exists a minimal model that contains no $A_{j}, 1 \leq j \leq l$, then the proof is similar to case 1 . Second, if originally $E_{D B} \vdash A_{1} \vee \cdots \vee A_{l}$, then $A_{1} \vee \cdots \vee A_{l}$ now must be deleted. The algorithm replaces $A_{1} \vee \cdots \vee A_{l}$ by $A_{1} \vee \cdots \vee A_{l} \vee B$, for all possible ground atoms $B$ not in the disjunction. Clearly this is minimal (see Deletion 2 algorithm) and conditions 1 and 2 are satisfied.

Case $3 . C_{j}$ is positive. Same argument as for the second part of case 2.

\section{Conclusion}

We have developed algorithms for the insertion and deletion of intensional atoms into and from stratified disjunctive databases. We have also shown in what sense these algorithms are optimal. There are a variety of ways in which this work can be extended. For example, it would be useful to consider Skolem constants as in [8] to apply this approach to programs with variables; and it may be possible to use the tree representation of the minimal models of a disjunctive database described in [3] to implement the insertion of information into the stratified database. Such algorithms are currently under investigation. The most technically challenging project, however, would be to extend the algorithms developed here to a richer class of disjunctive databases which are not stratified, and also rules that contain disjunctions in their heads.

\section{Acknowledgement}

We wish to thank Jose Alberto Fernandez, Chiaki Sakama, and the referee for helpful comments. 


\section{References}

[1] K.R. Apt, H.A. Blair and A. Walker. Towards a theory of declarative knowledge. In Foundations of Deductive Databases and Logic Programming, J. Minker (ed.), Morgan Kaufmann Publishers (1987), pp. 89-148.

[2] R. Fagin, J. Ullman, and M. Vardi. On the semantics of updates in databases. In Proceedings of the Second ACM Symposium on the Principles of Database Systems (1983), pp. 352-365.

[3] J. Fernandez and J. Minker. Bottom up evaluation of disjunctive deductive databases. Submitted for publication.

[4] A. Guessoum and J. Lloyd. Updating knowledge bases. New Generation Computing 8(1990), pp. 71-89.

[5] A. Guessoum and J. Lloyd. Updating knowledge bases II. New Generation Computing 10(1991), pp. 73-100.

[6] J. Minker. On indefinite databases and the closed world assumption. In Lecture Notes in Computer Science 138, Springer Verlag (1982), pp. 292-308

[7] R. Reiter. On closed world data bases. In Logic and Data Bases, H. Gallaire and J. Minker (eds.), Plenum Publishing Corporation (1978), pp. 119-140.

[8] F. Rossi and S. Naqvi. Contributions to the view update problem. In Proceeding of the Sixth International Conference on Logic Programming (1989), pp. 398-415. 